\title{
A Survey on usage of Ontology in Different Domains
}

\author{
N. Madurai Meenachi \\ Scientific Information Resource Division, \\ Resources Management Group, \\ Indira Gandhi Centre for Atomic Research, \\ Kalpakkam - 603102 \\ Tamil Nadu, India
}

\author{
M. Sai Baba \\ Scientific Information Resource Division, \\ Resources Management Group, \\ Indira Gandhi Centre for Atomic Research, \\ Kalpakkam - 603102 \\ Tamil Nadu, India
}

\begin{abstract}
A survey has been presented on the usage of ontology in various domains like Medical, Agriculture, Geosciences, Education, Marine, Communication, Computer, Chemical, Defence, Linguistic etc. A summary of the available ontology developed in various domains is given and no attempt has been made to evaluate them. Only a broad picture of ontology applications in various domains practiced today are described. In some cases details like number of concepts, relationship, classes and subclasses defined are also given. The survey indicated that considerable effort has gone in the development of ontology in the domains of medical, education, computer science. It is noted that rather limited effort has gone into the development of ontology in the domains of power plants and atomic energy.
\end{abstract}

\section{General Terms}

Ontology Survey

\section{Keywords}

Semantic web, Ontology, web ontology language (OWL).

\section{INTRODUCTION}

Ontology is an explicit formal specification of the terms in the domain and relations among them [1]. Ontology is used to capture the knowledge of any particular domain to avoid ambiguity of terms. The major advantage of use of ontology is that it will provide a globally unique identifier for all concepts. It is used to capture knowledge in any given domain. Ontology describes the concepts in a given domain and the relationships that hold between them. It helps to share common understanding of the structure of information among the users, to enable reuse, analyze the domain knowledge. It enables to merge already existing knowledge there by expanding it further [2]. Ontology for any domain is developed by formulating a set of questions that the envisioned knowledge-based agent should be able to answer [3]. Based on these questions, one identifies some of the concepts, sub concepts, relationships, features and instances that are defined as the part of the ontology. While sharing the domain knowledge, ontology is used to make the knowledge interoperable and also reusable thereby having seamless exchange of information among the applications [4]. Ontology is developed to provide the common semantics for agent communication so that it acts as a bridge when two or more agents need to communicate or exchange information [5]. In any domain, controlled vocabulary of words from that domain is taken for knowledge representation. A controlled vocabulary is a set of restricted words, used for describing resources or discovering data for any domain. When representing knowledge for the domain, controlled vocabulary prevents misspellings and avoids the use of arbitrary, duplicate or perplex words that cause inconsistent knowledge. Ontology is implemented in almost all fields of study like medical, aviation, computer science, commerce, mechanical engineering, space, food, agriculture, linguistics etc. A survey was undertaken to list out the fields where the domain ontology is established.

At our Centre, we have initiated a program to develop ontology application for Nuclear Energy Domain. In that context a survey of literature was carried out which indicated that ontology has been defined and applied in various domains. A survey of using of ontology in various domains is given in this paper.

The survey of ontology application in various domains was based on the available research papers, referred journals, reports in the respective domains, scholarly articles available on the advantages of ontology application and usage. This survey has been carried out in domains like agriculture, education, medicine, defense, nuclear power where the usage of ontology is proved to be extremely helpful. Only a broad picture of ontology applications in various domains practiced today are surveyed and described. No attempt has been made to evaluate them. The Domains surveyed are listed in alphabetical order. Since the impact of ontology in various domains is very large, we have not attempted the survey to be exhaustive.

\section{ONTOLOGY DEVELOPED IN THE DOMAIN OF AGRICULTURE}

AGROVOC is used for agriculture controlled-vocabulary searching in various systems throughout the Food and Agriculture Organization (FAO) [6]. It consists of organized terms with multi languages support including the terminology of agriculture, forestry, fisheries, food and other related domains in agriculture [7]. Thereby accessing the structure and standardize agricultural terminology in multiple languages by the system and the users [8].

FSSRS (Food Safety Semantic Retrieval System) is an ontology-based semantic retrieval experimental system, includes all aspects of food safety knowledge in the field of 
emergencies. This system provides the users to access the accumulation of the knowledge in the food safety domain [9].

According to Ling cao et al, Agriculture Literature Retrieval System defined agriculture literature concepts captured from Encyclopedia of Chinese Agriculture and Catalogue of Ancient Chinese Agricultural Literatures. There are more than 10,000 keywords extracted from the research papers of Chinese agricultural history [10].

Citrus Water and Nutrient Management System (CWMS) is defined for Water and nutrient balance processes for citrus production having 700 symbols and 500 equations. This includes concepts like block, soil cell, soil profile, soil layer, root distribution, irrigation system, and weather [11]

OntoSim-Sugarcane is an application which represents hydrology, nutrient cycling, plant growth, soil moisture, crop growth on organic soils and nutrient uptake in southern Florida sugarcane production. This collection consists of 195 equations and 247 symbols [12].

\section{ONTOLOGY DEVELOPED IN THE DOMAIN OF AVIATION}

In Civil Aviation domain, concepts and role are defined as Aircraft, Non_aircraft, Emergency_in_aircraft, Emergency_in_non_aircraft etc. Here the ontology is described using description logic language which facilitates for checking of rationality by defining concepts, roles and instances [13].

\section{ONTOLOGY DEVELOPED IN THE DOMAIN OF BIOLOGY}

Plant Ontology Database describes the controlled vocabulary (ontology) for plants. It is a collaborative effort among model plant genome database developer and plant researchers to create and maintain the database. It also implements a semantic framework to make meaningful crossspecies and database comparisons [14]. Plant Anatomy consists of 30087 terms defining a controlled vocabulary of plant's morphological and anatomical structures representing organs, tissues, cell types and their biological relationships based on spatial and developmental organization example stamen, gynoecium, petal, parenchyma, guard cell and plant structure. In Plant Structure development stage consists of a controlled vocabulary of growth and developmental stages in various plants and their relationships [15].

Plants Domain Ontology has the collection of botany knowledge and environment knowledge. The botany knowledge consists of knowledge of geometry and topology [16].

TAMBIS (Transparent Access to Multiple Bioinformatics Information Sources) uses ontology to enable biologists to retrieve knowledge by querying the multiple external databases [17]. It has approximately 400 classes and 100 object properties.

BRENDA (BRaunschweig ENzyme DAtabase) is maintained at the Technology University, Germany. It allows the users to search simultaneously in all biochemically relevant ontology. It contains approximately 3400 terms on tissues, organs and cell types. FRENDA (Full Reference Enzyme DAta) and AMENDA (Automatic Mining of Enzyme DAta) are additional databases created by text-mining procedures. Biochemical and molecular properties of enzymes such as classification and nomenclature, reaction and specificity, functional parameters, organism-related information, enzyme structure, isolation and preparation, literature references, application and engineering, enzyme-disease relationships are also available. BRENDA contains ontology definition for 3300 enzymes in 500 different organisms [18].

\section{ONTOLOGY DEVELOPED IN THE DOMAIN OF CHEMISTRY}

In the chemistry domain, ontology has two main categories: Chemical-Elements and Chemical-Crystals. ChemicalElements have 16 concepts, 103 instances, 3 functions, 21 relations and 27 axioms. Whereas Chemical-Crystals have 19 concepts, 66 instances, 8 relations, and 26 axioms. Chemicals also include public Ontolingua ontologies, such as StandardUnits, Standard-Dimensions, and Knowledge interchange format lists [19].

Chemical Entities of Biological Interest (ChEBI) is a dictionary of molecular entities in the 'small' chemical compound. Number of entities in ChEBI is approximately 18000 , whereby the relationships between molecular entities or classes of entities and their parents and children are specified [20].

\section{ONTOLOGY DEVELOPED IN THE DOMAIN OF CIVILENGINEERING}

Healthy Housing is an important concept on construction field. It defines the regulations of vocabularies characterizing the residency from the knowledge of healthy housing, creating the terminologies and relations for the core vocabularies of the field. OWL language is used for defining these terminologies and relations [21].

National Semantic Web Ontology project in Finland (FinnONTO) was taken up to try and build a national infrastructural foundation for the semantic web. This is carried out by establishing a large research consortium representing universities, public organizations, companies and working in collaborative mode [22].

\section{ONTOLOGY DEVELOPED IN THE DOMAIN OF COMPUTER SCIENCE}

The ITiCSE(Innovation and Technology in Computer Science Education) 2007 Computing Ontology describes various disciplines, topics, subtopics that belong to the domain of Computer Sciences. It is a collection of computing topics, early methods, devices, machines, hardware (nonsystem), hardware-software systems, software, theory, people, institutions and milestones [23].

SOUPA (Standard Ontology for Ubiquitous and Pervasive Applications) is designed to model and support pervasive computing applications. Ontology is expressed using the Web ontology language OWL. It includes modular component vocabularies to represent intelligent agents with associated beliefs, desires and intentions, time, space, events, user profiles, actions and policies for security and privacy [24].

Software system ontology is a combination of domain ontology and class diagram ontology. Domain ontology is domain vocabulary which is built by domain experts, while class diagram ontology is automatically populated from source code to represent the knowledge in the code. It also includes method of class diagram to ontology transformation and algorithm of ontology combination [25]. 
Software product management domain aims to identify the recent domain-specific research on software product management. It extracts text corpus with respect to terms, concepts, hierarchical relations of the concepts and the nonhierarchical relationships between the concepts used for ontological learning process [26].

Open Mind Indoor Commonsense (OMICS) is a collection of commonsense data consisting of 152098 items by 1009 users for indoor mobile robots [27].

Video Indexing and Retrieval consists of two types of ontology namely Object Ontology and Shot Ontology. In Object Ontology users are allowed to query a video collection using semantically meaningful concepts without the need for performing manual annotation of visual information. But in Shot Ontology users are allowed to retrieve the video by submitting either single or multiple keywords queries [28].

In the Software Engineering Lifecycle, ontology is defined for each phase from Analysis, design, requirement engineering, component reuse, implementation, integration, testing till documentation [29].

In Telecommunication Management Network model, semantic is introduced to fix the interoperability problem of the network and its equipment. In the domain of network ontology, the concepts like tangible router interface, intangible border gateway protocol parameters, network objects and management operations are defined [30].

Image Classification Using Neural Networks is used to classify objects from an image. The network takes an image as input and gives classification as output which is processed by ontology to discover the relationships among objects [31].

\section{ONTOLOGY DEVELOPED IN THE DOMAIN OF E-BUSINESS}

SymOntoX (Symbolic Ontology XML-based management system), is a software for the OntoPrivacy, BEO (Business and Enterprise Ontology) and IMHO (Interoperable Minimal Harmonise Ontology). OntoPrivacy supports a legal XML database for the protection of personal information. BEO represents the core of an ontology-based platform for Business Games. IMHO is tourism ontology developed in the Harmonise Project. SymOntoX has added advantage of multi lingual support [32].

\section{ONTOLOGY DEVELOPED IN THE DOMAIN OF EDUCATION}

Sharable Content Object Reference Model (SCORM) is a collection of standards and specifications for web-based elearning technology [33].

TM4L (Topic Maps for e-Learning) provides support for creating and using ontology-aware topic maps-based repositories of online materials. It includes research papers, special journal issues, books, projects, software, conferences, workshops, mails, research labs, working groups and people having a unique Uniform Resource Identification [34].

In Web-Based Educational Systems (WBES), defines communication ontology, communication content ontology and interaction protocol ontology [35].

Ontologies for Education (O4E) consists of concepts like technological perspective, application perspective. Technological perspective defines the knowledge organization, knowledge inference, information, information vizualisation, information navigation, information querying, subject domain ontology, instructional knowledge. Application perspective defines sub concepts in knowledge construction, knowledge externalization, knowledge communication, architectural knowledge [36].

OURAL (Ontologies for the Use of digital learning Resources and semantic Annotations on Line) defines ontology in the elearning domain which includes problem-situation, problem solving, critical analysis, case study, debate, cyber quest, project, exercise etc. The resources can be digital or non digital merge with part of the learning context [37].

Learning Resource metadata for the description of the content of learning resources provides a range of standard and universal method. An educational learning resource ontology semantic network defines concept as ontology learning resources, context, ontology, ontology learning resource structure [38].

European Credit Vocational System uses ontologies in the construction of the educational resources library, to provide a common access to the information regarding the qualification systems of nine European countries [39].

Semantic Web ontologies for e-learning systems in higher education define educational ontology consisting of user profile ontology, the person ontology, the contact ontology and the activities ontology. This was implemented using Protégé-OWL ontology editor [40].

The Dutch system of Secondary Vocational Education, which entails ISCED-97 (International Standard Classification of Education) educational levels 3 and 4, is designed to train students of age 14 to 18 and adults for professions at ISCO88(International Standard Classification of Occupations) skill level 2. Using Protégée ,the ontology of core duty, specific information, core part, qualification profile, competence, core assignment, certifiable unit, criteria for mastery, exit differentiation etc. are defined [41].

National School of Commerce and Management defines Ontologie_US_ENCG which consists of administrative documents, project documents, financial information, legislation, institutional life, procedures, pedagogical document, exploration, capitalization, management, exam, meeting reports, general policy classes. Protégé 2000 editor was used for development [42].

The Virtual Lab Ontology has been developed using Protégé. Ontology "VLabResources" is defined to include all resources needed for any practical activity in an engineering education program. The classes like subjects, competence and tasks are defined to perform the practical activities of the Virtual Lab in a virtual learning environment. They have used standard reasoner tools like Pellet or $\mathrm{FaCT}++$ for validation [43].

Economic ontologies proposed to represent the structure of economic knowledge in Croatia to define taxonomy of economics. It represents institutional curricula, academic discipline, documenting the data and metadata, meta data about learning and management systems, online resources for training materials and teaching [44].

Cultural Artefacts in Education (CAE) ontology for different country like China, UK and Ireland consists of interrelated sub-ontologies, authority, group language, lesson and data [45]. 
Sahayika is used for building knowledge structures in education domain in India and its interface is also available in English and Bengali language. This deals with school education domain which covers subjects like biology, geography, physics, chemistry, history etc. [46].

Remote education system classified as teaching practice and teaching management. Teaching practice ontology covers course browse, learning, online examination and online direct learning of interested parties, electronic courseware management etc. [47].

The purpose of the Information and Communication Technologies (ICT) Education Ontology is to provide a central repository of classified knowledge in ICT education. ICT ontology consists of concepts like Ontology of ICT curriculum, ontology of ICT Job, ontology of ICT Skill and ontology of ICT Research [48].

\section{ONTOLOGY DEVELOPED IN THE DOMAIN OF ELECTRONICS}

A Suggested Upper Merged Ontology (SUMO) defines ontology for sensor networks domain and link them through the upper SUMO ontology. It defines approximately 25,000 terms and 80,000 axioms about CPU processing power, memory, power supply, and radio and sensor modules [49]. The SUMO ontology comprises various domains such as computing services (networks, systems, and services), finance, geography, time, economy and transportations [50].

\section{ONTOLOGY DEVELOPED IN THE DOMAIN OF GEOSCIENCE}

SWEET (Semantic Web for Earth and Environmental Terminology) is a project by NASA for developing domain ontologies to describe earth science data and knowledge. It includes the Earth Realm, Non-Living Element, Living Element, Physical Property, Units, Numerical Entity, Temporal Entity, Spatial Entity, Phenomena and Human Activities ontologies [51]. There are 6000 concepts in 200 separate ontologies defined in SWEET [52].

Earth and Planetary ONTology (EPONT) is a domain level ontology for sharing data among geoscientists. It uses existing community-accepted high level ontologies such as SUO (Semantic Upper Ontology: IEEE endorsed), SWEET (Semantic Web for Earth and Environmental Terminology) and NADM (North American Geological Data Model) [53].

In Geoscience domain, FGDC (Federal Geographic Data Committee) content standard for digital geospatial metadata was developed to describe all possible geospatial data [54].

The Bremen University Semantic Translator for Enhanced Retrieval combines ontology-based metadata with an ontology-based search. This ontology is used to find the geographic information services for estimating potential storm damage in forests [55].

Geological hazard ontology, which is a hierarchical framework in geological hazard information, which can define the ontology concept of hazard geology, such as earthquakes, landslip, landslides, debris flow and other hazard [56].

\section{ONTOLOGY DEVELOPED IN THE DOMAIN OF HUMAN RESOURCES}

Ontology has been developed for existing human resources management standards and systems classifications like Compensation Ontology which is based on the ISO 4217 and there are 254 instances, Driving License Ontology, Economic Activity Ontology, Occupation Ontology based on the ISCO88 which has 609 concepts, Education Ontology, based on the ISCED and has 130 concepts, Geography Ontology based on the ISO 3166 and has 490 instances, Skill Ontology based on European Dynamics Skill classification and has 291 skills, Competence Ontology, Time Ontology based on DAML ontology [57].

\section{ONTOLOGY DEVELOPED IN THE DOMAIN OF LINGUISTIC DOMAIN}

Iban is one of the divergent Dayak ethnic groups in Sarawak. Sarawak is one of two Malaysian states on the island of Borneo. Iban WordNet (IbaWN) was developed using this domain ontology Using Iban as the main language, ontology for agricultural domain was constructed. [58].

SOLAT-based ontology involves the Al Qur'an, the authentic Hadith, and books that focus on the Shafie's school of thought. It involves the types and characteristics of Solat, hukm, purification such as ghusl, wudu and Tayammu. It also includes Qurani verses in Arabic language, images and video. There are 48 concepts, 51 properties and 282 instances [59].

Chinese ancient poetry learning system provides the high knowledge relevance among poems, poet, allusion, genre etc., and presents knowledge according to the user's preference and educational level. The system collects about 270000 of ancient poems and 10000 of allusions [60].

ENGOnto, integrates multiple relevant ontologies for personalized agents to deal with dynamic changes of learner's learning process, interaction between instructor and learner and learning resources in the environment of English language education. This ontology consists of People Ontology, Language Ontology, Pedagogy Ontology, Curriculum Ontology and Knowledge-Point Ontology, for individual English personalized learning [61].

\section{ONTOLOGY DEVELOPED IN THE DOMAIN OF LIBRARY}

Ontology based Chinese digital library resources consist of Ontology of bibliographic relations, Ontology-based digital library metadata schema, MARC format and thesaurus. It also involves mapping data from MARC to the ontology, and reasoning about the data to establish the relationships [62].

Document classification system (DCSO) consists of four modules: Keyword Extraction, Ontology Construction, Document Classification and Document Searching. In this system formal concept analysis (FCA) method is used for the analysis of data. Nearly 525 documents in the area of information management are retrieved from the Electronic Theses and Dissertations System. Amongst these, 360 documents act as the training document and 165 documents for testing purpose [63].

\section{ONTOLOGY DEVELOPED IN THE DOMAIN OF MARINE SCIENCES}

Marine Metadata Interoperability (MMI) develops a web based marine metadata vocabularies for the users [64].

Maritime Domain Awareness (MDA) integrates information from multiple sources in a complex and evolving scenario to produce a dynamic, comprehensive, and accurate picture of the naval operations environment. This would aid in identification of suspicious ships of interest [65]. 
There exist approximately 200000 kinds of marine life. By Marine Biology ontology, ocean experts can extract relevant knowledge about oceanic food chain and biodiversity protection. Marine biology ontology include concepts like halobios, plankton, neuston, nekton, benthos, phytoplankton, zooplankton, bacterioplankton, pleuston, epineuston hypoeneuston, vertebrates, molluscs and crustaceans, zoobenthos, phytobenthos etc. Approximtely 160 terms are available in this domain [66].

\section{ONTOLOGY DEVELOPED IN THE DOMAIN OF MATHEMATICS}

Semantic relatedness measures the closeness or likeness between concepts in natural language processing. It has been implemented in lexical ontologies such as WordNet. The important features of this method include a unique approach to the weighted edge measure. Each edge is weighted based on applying a concept probability algorithm to a multiset composed of ontology property ranges [67].

Open Mathematical Document (OMDoc) is used as an ontology language. It is a content-based markup which focuses on the semantic mathematical formulae. Learning Style Ontology consists of two main components, namely cognitive processing and modality perception. Cognitive processing includes two attributes like analytical and global whereas modality perception comprised of four attributes like visual, verbal, auditory and tactile-kinesthetic [68].

\section{ONTOLOGY DEVELOPED IN THE DOMAIN OF MEDICINE}

The Gene Ontology contains structured, controlled vocabularies and classifications for several domains of molecular and cellular biology and is freely available in the annotation of genes, gene products and sequences. It has 22684 biological_process, 2987 cellular_component and 9375 molecular_function [69].

UBERON is a multi-species metazoan anatomy ontology. This is created to support translational research by allowing comparison of phenotypes across species and provide logical cross-product definitions for Gene Ontology biological process terms. The current version of the ontology has 2808 terms, 5110 links between terms, 9339 links out to other anatomical ontologies, more than 1643 Wikipedia crossreferences and has been referenced in 682 Gene Ontology cross-products [70].

The MGED Ontology (Microarray Gene Expression Data Ontology) defines all aspects of a microarray experiment. It also analyzes the data to describe the design of the experiment and array layout, through the preparation of the biological sample and the protocols used to hybridize the RNA (Ribonucleic acid). There are 233 Classes, 143 properties and 681 individuals defined in this ontology [71].

Mouse Genome Database (MGD) a model for studying human biology and disease integrates genetic, genomic and phenotypic information about the laboratory mouse. It also includes comprehensive characterization of genes and their functions, standardized descriptions of mouse phenotypes, extensive integration of DNA and protein sequence data, normalized representation of genome and genome variant information including comparative data on mammalian genes [72].
Clinical Bioinformatics Ontology (CBO), a semantic network describes clinically significant genomics concepts. It also includes concepts appropriate for both molecular diagnostics and cytogenetic [73]. It contains approximately 8155 concepts, 18946 relationships, 4304 facets and 13341 terms.

Thoracic Radiology contains knowledge of anatomy and imaging procedures. In this a total of 138 classes, including radiology orderable, procedures, procedure steps, imaging modalities, patient positions, and imaging planes. Radiological knowledge was encoded as relationships among these classes [74].

SNOMED-CT (Systematized Nomenclature of Medicine-Clinical Terms) is a terminology system developed by the College of American Pathologists. It contains over 344,000 concepts and was formed by restructuring of SNOMED RT (Reference Terminology) and the United Kingdom National Health Service Clinical Terms [75].

UMLS (Unified Medical Language System) is a repository of biomedical vocabularies developed by the US National Library of Medicine. The UMLS integrates over two million names for some 900,000 concepts from more than 60 families of biomedical vocabularies, as well as twelve million relations among these concepts [76].

GoMiner is an application that organizes lists of under and over expressed genes from a microarray experiment for biological interpretation in the context of the Gene Ontology. GoMiner achieves a computational resource that automates the analysis of multiple microarrays and integrates results across all of the microarrays [77].

The FungalWeb Ontology supports the data integration needs of enzyme biotechnology from inception to product roll out. It serve as a knowledge base for decision support, to link fungal species with enzymes, enzyme substrates, enzyme classifications, enzyme modifications, enzyme retail and applications [78]. It contains 3667 concepts, 12686 instances and 157 Properties [79].

Protein Mutation Impact Ontology conceptualizes impacts and the mutations associated with them. To design the Mutation Impact Ontology, information Text elements, biological entities and entity relations are also required. Using OWL the relations between these entities are expressed [80].

PIPS (Personalized Information Platform for Health and Life Services) deals with medical knowledge, food and nutrition knowledge, about patients, and their clinical records, products and treatments. In PIPS project, the Food Ontology deals with the development process that describes 177 classes, 53 properties and 632 instances [81].

Neuro-pediatric Physiotherapy is an area that includes diagnosis, treatment and evaluation of babies by the physiotherapist in order to observe the progress of treatment. Neuro-pediatric Ontology is composed by 100 classes and subclasses, 30 properties and 200 axioms [82].

Cardiovascular Medicine Ontology in the domain of Mechanical Circulatory Support Systems (MCSS) is designed to avoid the lack of uniformity in the information available in the field. Ontology is a catalogue of existing items with 30 different types [83].

Cystic Fibrosis is a subset extracted from a large MEDLINE (Medical Literature Analysis and Retrieval 
System Online) collection. There are 1239 files with 821 concepts and average number of concepts assigned to a document is 2.8 [84].

The National Center for Biomedical Ontology (NCBO) maintains BioPortal, an open library of more than 200 ontologies in biomedicine. The aim of this portal is to provide a researcher to browse and analyze the information stored in these diverse resources [85].

The National Cancer Institute (NCI) Thésaurus is a public domain description logic-based terminology for Bioinformatics caCORE distribution. As on 2003 release, it contains 26,000 concepts and 71,000 terms divided among 24 taxonomies. The final OWL ontology is made up of approximately 450,000 triples in a file that is over 33MB [86].

The Foundational Model of Anatomy Ontology (FMA) is a domain ontology that represents a coherent body of explicit declarative knowledge about human anatomy. It is a framebased ontology and there are 148 relationship types, 70,000 anatomical concepts interrelated by over 580,000 relationship instances [87].

\section{ONTOLOGY DEVELOPED IN THE DOMAIN OF MILITARY}

In the military intelligence domain ontology are referred the ontology structure in HowNet and WordNet, and it stores the characteristics of the military information [88].

5W1H-based conceptual modeling framework for domain ontology is proposed, which is used to analysis domain concepts and relations from six aspects including Who, When, Where, What, Why and How. According to this framework, the conceptual model of Science and Technology Project Ontology (STPO) in science and technology domain is designed. From the analysis, real world model is designed using the $5 \mathrm{~W} 1 \mathrm{H}$ conceptual modeling framework by mapping the class model in the Object-Oriented method [89].

Collaboration of MDOCA (military domain ontology construction approach), SOCA (situation ontology construction approach) and MROCA (military rule ontology construction approach) are used to construct SO (situation ontology) and MRO (military rule ontology) [90].

\section{ONTOLOGY DEVELOPED IN THE DOMAIN OF NUCLEAR WEAPONS}

In Nuclear Weapon domain, radionuclide concepts are defined. It has sub concepts like data products, laboratory managers, programs, facilities and data managers [91].

\section{ONTOLOGY DEVELOPED IN THE DOMAIN OF NEWS}

In the News domain, information extraction doesn't rely on the page structure but the result of this information extraction cooperates with the pre-defined Ontology. The web pages downloaded with the use of .NET's Web Browser component are formed into a DOM (DOcument Modeling) tree. Ontology of news domain consists of following sub concepts like navigation page, seed page, content page, navigation page marker path, content page marker path, title, time, picture and content [92].

\section{ONTOLOGY DEVELOPED THE DOMAIN OF POWER PLANTS}

A Safety Assessment Management Information System for Power Plants based developed on a client server model. It has been used in power plant of Datang Group Corporation in China and reported to be satisfactory. Knowledge of equipment fault was captured by knowledge transformation, collecting original literature and data, identifying relations among basic glossaries which contain complex information, determining the rules [93].

Steam Turbine (ST) ontology is created by integrating and merging with existing databases. It enables sharing the knowledge through a shared ontology for the maintenance of a steam turbine [94].

\section{ONTOLOGY DEVELOPED IN THE DOMAIN OF TRANSPORT}

The main components of the pallet transfer system are conveyor belts which deliver items from one place to another, index stations, identification units (RFID) for identification of passing pallet units, and intersection units. This is used in ontology to represent locations whose attributes provides the details of locations reachable by it [95].

\section{SUMMARY}

A survey on usage of ontology in different domains is given in this paper. The survey is based on the available literature and not attempted to be either exhaustive or complete. No attempt has been made to evaluate them. It can be seen from the survey that ontology has been applied in several domains encompassing agriculture, education, medicine, defense, aviation, computer science and linguistics etc. From this survey it can be inferred that extensive work has been reported in the medical domain. Work has been done in defining ontology from micro organism to macro organism. The ontology development covers concepts related to database of patients and diseases with multilingual support. This would pave the way to share expert medical knowledge without any boundaries. In the domain of education, efforts have been towards creating systems that aid learning process along with formal teaching. From kindergarten to higher education, ontology has been extensively defined to achieve person independent knowledge based system. In the computer domain, the ontology development has evolved considerably, in defining concepts relating to hardware and software systems, image processing, videos and audios and neural networks.

Enhanced development of ontology would aid in the evolution of semantic web leading to complete sharing of knowledge in a given domain. It is also to be inferred that the ontology development is a continuous process and success could be achieved by participation of domain experts and users. The survey indicated that in some domains like power plants, atomic energy, the ontology development has been rather limited. We have initiated a program to develop ontology based knowledge representation in the domain of nuclear power plants. This study aided us to get the overall idea of domain concepts implementation and tools required for the development.

\section{ACKNOWLEDGEMENTS}

It is a pleasure to acknowledge the encouragement of Shri S.C. Chetal, Director, IGCAR. We also acknowledge the colleagues of SIRD, RMG for their participation and support. 


\section{REFERENCES}

[1] Gruber, T.R. 1993. A Translation Approach to Portable Ontology Specification Knowledge Acquisition.199-220.

[2] Michael Grüninger., Steven Shapiro., Mark S. Fox., and Harald Weppner. 2011. "Combining RFID with ontologies to create smart object", International Journal of Production Research,Taylor and Francis. 1-23.

[3] Gruninger, M. and Fox, M.S. 1995. Methodology for the Design and Evaluation of Ontologies. In Proceedings of the Workshop on Basic Ontological Issues in Knowledge Sharing.

[4] Natalya F. Noy. and Deborah L. McGuinness.2001. Ontology development 101 a guide to creating your first ontology,http://protege.stanford.edu/publications/ontolog y_development/ontology101-noy-mcguinness.html

[5] Ding, Y. and Foo, S. 2002. "Ontology Research and Development Part 2 - A review of ontology mapping and evolving", Journal of information science, 375-388.

[6] Dagobert Soergel., Boris Lauser., Anita Liang., Frehiwot Fisseha.,Johannes Keizer., and Stephen Katz.2004. "Reengineering Thesauri for New Applications: the AGROVOC Example", Journal of Digital Information. $1-23$.

[7] Salvador Sánchez-Alonso. and Miguel-Angel Sicilia. 2009. Using an AGROVOC-based ontology for the description of learning resources on organic agriculture, Metadata and Semantics, Springer. 481-492.

[8] Margherita Sini., Sachit Rajbhandari., Maziar Amirhosseni., Gudrun Johannsen., Ahsan Morshed., and Johannes Keizer. 2012. The AGROVOC Concept Server Workbench System: Empowering management of agricultural vocabularies with semantics Scientific and Technical Information and Rural Development. In Proceedings of IAALD XIIIth World Congress, Montpellier.

[9] Yuehua Yang., Junping Du., and Meiyu Liang. 2011. Study on food safety semantic retrieval system based on domain ontology. In Proceedings of IEEE Cloud Computing and Intelligence Systems. $40-44$.

[10] Ling Cao. and Lin He. 2008. Domain Ontology-based Construction of Agriculture Literature Retrieval System. In Proceeding of $4^{\text {th }}$ International Conference on Wireless Communications, Networking and Mobile Computing , 1- 3 .

[11] Howard Beck., Kelly Morgan., Yunchul Jung., Sabine Grunwald., Ho-young Kwon., and Jin Wu. 2010. Ontology-based simulation in agricultural systems modeling, Agricultural Systems, Elsevier. 463-477.

[12] Ho-Young Kwon., Sabine Grunwald., Howard W. Beck., Yunchul Jung., Samira H Daroub., Timothy A. Lang., and Kelly T. Morgan. 2010. Ontology-based simulation of water flow in organic soils applied to Florida sugarcane, Agricultural Water Management, Elsevier. $112-122$

[13] Wang Hong., Gao Siting., and Wang Jing. 2011. The Application Research of Description Logic in Civil Aviation Domain Ontology. In Proceedings of
International Conference on Management and Service Science, $1-4$.

[14] Shulamit Avraham., Chih-WeiTung., Katica Ilic., Pankaj Jaiswal., Elizabeth A. Kellogg., Susan McCouch., Anuradha Pujar., Leonore Reiser,Seung Y Rhee., Martin M Sachs., Mary Schaeffer., Lincoln Stein,Peter Stevens., Leszek Vincent., Felipe Zapata., and Doreen Ware. 2008. "The Plant Ontology Database: a community resource for plant structure and developmental stages controlled vocabulary and annotations", Nucleic Acids Research, Oxford University Press. D449-D454.

[15] Pankaj Jaiswal., Shulamit Avraham., Katica Ilic., Elizabeth A Kellogg., Susan McCouch., Anuradha Pujar., Leonore Reiser., Seung Y Rhee., Martin M. Sachs., Mary Schaeffer., Lincoln Stein., Peter Stevens., Leszek Vincent., Doreen Ware., and Felipe Zapata. 2005. "Plant Ontology (PO): a controlled vocabulary of plant structures and growth stages", Comparative and Functional Genomics.388-397.

[16] Fan Jing., Zhang Xin-pei., and Dong Tian-yang. 2008. Research of Plant Domain Knowledge Model Based on Ontology, In Proceedings of IEEE 3rd International Conference on Innovative Computing Information and Control.

[17] Goble, C. A., Stevens,R., Ng,G., Bechhofer, S.,Paton, N. W., Baker, P. G., Peim, M., and Brass, A. 2001. "Transparent access to multiple Bioinformatics information sources”, IBM Systems Journal, 532-551.

[18] Antje Chang., Maurice Scheer., Andreas Grote., Ida Schomburg., and Dietmar Schomburg., 2009. "BRENDA, AMENDA and FRENDA the enzyme information system: new content and tools", Nucleic Acids Research, Oxford University Press, D588-92.

[19] Fernández López., Asunción Gómez-Pérez., and Juan Pazos Sierra. 1999. "Building a Chemical Ontology Using Methontology and the Ontology Design Environment, Mariano", IEEE Intelligent Systems. IEEE, 37-46.

[20] Paula de Matos.,Rafael Alcántara., Adriano Dekker., Marcus Ennis., Janna Hastings., Kenneth Haug., Inmaculada Spiteri.,Steve Turner., and Christoph Steinbeck. 2010. "Chemical Entities of Biological Interest: an update", Nucleic Acids Research. Oxford University Press, D249-D254.

[21] Yi Zhang Li., Li Tan., Jie Liu., and ChongChong Yu. 2010. A Domain Ontology Construction Method Towards Healthy Housing. In Proceedings of 2nd International Conference on Information Engineering and Computer Science.

[22] Eero Hyvonen. 2006. FinnONTO_Building the Basis for a National SemanticWeb Infrastructure in Finland. In Proceedings of the $12^{\text {th }}$ Finnish AI Conference STeP.

[23] Lillian N. Cassel., Gordon Davies., William Fone., Anneke Hacquebard., John Impagliazzo., Richard LeBlanc., Joyce Currie Little., Andrew McGettrick., and Michela Pedrona. 2007. The computing ontology: application in education. In Proceedings of $38^{\text {th }}$ SIGCSE technical symposium on Computer science education. 
[24] Perich, F., Finin, T., and Joshi, A. 2004. SOUPA: Standard ontology for ubiquitous and pervasive applications, 2004. In Proceedings of the $1^{\text {st }}$ Annual International Conference on Mobile and Ubiquitous Systems: Networking and Services. 258 - 267.

[25] HongZhou., FengChen., and HongjiYang. 2008. Developing Application Specific Ontology for Program Comprehension by Combining Domain Ontology with Code Ontology, In Proceedings of the $8^{\text {th }}$ International Conference on Quality Software, 225-234.

[26] Botzenhardt., Alexander Maedche., Juri Wiesner., Developing a Domain Ontology for Software Product Management, 2011. In Proceedings of $5^{\text {th }}$ International Workshop on Software Product Management, 7-16.

[27] Dongyeop Kang., Eugene Seo., Sookyung Kim., and HoJin Choi. 2009. Automatically Learning Robot Domain Ontology from Collective Knowledge for Home Service Robots, In Proceedings of $11^{\text {th }}$ International Conference on Advanced Communication Technology, 1766-1771.

[28] Vasileios Mezaris., Ioannis Kompatsiaris., Nikolaos V. Boulgouris., and Michael G. Strintzis. 2004."Real-Time Compressed-Domain Spatiotemporal Segmentation and Ontologies for Video Indexing and Retrieval, Circuits and Systems for Video Technology", IEEE Transactions, $606-621$.

[29] Hans-Jorg Happel., and Stefan Seedorf. 2006. Applications of Ontologies in Software Engineering, In Proceedings of $2^{\text {nd }}$ International Workshop on Semantic Web Enabled Software Engineering .

[30] Alfred Ka Yiu Wong., Pradeep Ray., Parameswaran, N., and John Strassner. 2005. "Ontology Mapping for the Interoperability Problem in Network Management", IEEE journal on Selected Areas in Communications, $2058-2068$.

[31] Casey Breen., Latifur Khan., and Arunkumar Ponnusamy. 2002. Image Classification Using Neural Networks and Ontologies. In Proceedings of IEEE conference on Database and Expert Systems Applications, 98-102.

[32] Missikoff, M. and Taglino, F. 2003. SymOntoX: a Webontology tool for ebusiness domains. In Proceedings of Fourth International Conference on Web Information Systems Engineering. $343-346$.

[33] Jin Tan Yang., Min Jey Hwang., and Yuan Fong Chu. 2005. A Study on Searching and Recommending SCORM CPs by Ontological Support. In Proceedings of the Fifth IEEE International Conference on Advanced Learning Technologies.

[34] Darina Dicheva., Sergey Sosnovsky., Tatiana Gavrilova., and Peter Brusilovsky. 2005. Ontological Web Portal for Educational Ontologies. In Proceedings of International Workshop on Applications of Semantic Web technologies for E-Learning.

[35] Darina Dicheva. and Lora Aroyo. 2004. A Minimalist Approach to Support Ontology-driven Educational Information Systems Communication. In Proceedings of International Workshop on Applications of Semantic.

[36] Lora Aroyo. and Darina Dicheva. 2004. "The New Challenges for E-learning: The Educational Semantic
Web". Journal of Educational Technology and Society, 59-69.

[37] Monique Grandbastien., Faiçal Azouaou., Cyrille Desmoulins., Richard Faerber., Dominique Leclet., and Céline Quénu-Joiron. 2007. Sharing an ontology in Education: Lessons learnt from the OURAL project. In Proceedings of $7^{\text {th }}$ IEEE International Conference on Advanced Learning Technologies.

[38] Dai Xiaopeng. and Li Xu. 2010. Study of Learning Source Ontology modeling in Remote education. In Proceedings of International Conference Multimedia Technology, 1-4.

[39] Feng Yang. and Youquan Chen. 2010. Ontology based application framework for Network Education Resources Library. In Proceedings of $2^{\text {nd }}$ International Workshop on Education Technology and Computer Science,423 - 426.

[40] Marian Bucos., Bogdan Dragulescu., and Marius Veltan. 2010. Designing a Semantic Web Ontology for Elearning in Higher Education. In Proceedings of IEEE $9^{\text {th }}$ International Symposium on Electronics and Telecommunications, $415-418$.

[41] ClaudioCubillos., FabrizioLamberti., and Claudio Demartini. 2008. Integration at Vocational Education and Training Level through Mapped Ontologies. In Proceedings of $3^{\text {rd }}$ International Conference on Convergence and Hybrid Information Technology,117122.

[42] Adil Laoufi., S.Mouhim., El Hassan Megder., and Chihab Cherkaoui. 2011. An Ontology Based Architecture to Support the Knowledge Management In Higher Education. In Proceedings of International Conference on Multimedia Computing and Systems, 1-6.

[43] Josep Prieto-Blázquez., Ivan García-Torà., Jordi HerreraJoancomartí., and Ana-Elena Guerrero-Roldán. 2008. Virtual Laboratory Ontology for Engineering Education. In Proceedings of $38^{\text {th }}$ ASEE/IEEE Frontiers in Education Conference, S2F-1 to S2F-6.

[44] Mesaric, J. and Dukic, B. 2007. An Approach to Creating Domain Ontologies for Higher Education in Economics.InProceeding of $29^{\text {th }}$ International Conference on Information Technology Interfaces, $75-80$.

[45] Stewart, C., Chandramouli. K., Cristea,A., Brailsford, T., and Izquierdo,E., 2008. Cultural Artefacts in Education: Analysis, Ontologies and Implementation. In Proceedings of International Conference on Computer Science and Software Engineering, $706-709$.

[46] Bhowmick, P.K., Bhowmick, S.,Roy, D., Sarkar, S., and Basu, A. 2007. Sahayika: A framework for participatory authoring of knowledge structures for education domain. In Proceedings of International Conference on Information and Communication Technologies and Development, $1-11$.

[47] Zhaoxu Wang., 2011. Research on Remote Education System Using Semantic Web. In Proceedings of International Conference on Computer Science and Network Technology, 2815- 2818.

[48] Kum Leng Chin. and Elizabeth Chang. 2011. A Sustainable ICT Education Ontology. In Proceedings of 
$5^{\text {th }}$ IEEE International Conference on Digital Ecosystems and Technologies, Korea, 350-354.

[49] Ian C Chow. and Jonathan J Webster. 2006. Mapping FrameNet and SUMO with WordNet Verb: Statistical Distribution of Lexical-Ontological Realization. In Proceedings of $5^{\text {th }}$ Mexican International Conference on Artificial Intelligence.

[50] Mohamad Eid., Ramiro Liscano., and Abdulmotaleb El Saddik. 2007. A Universal Ontology for Sensor Networks Data. In Proceedings of IEEE International Conference on Computational Intelligence for Measurement Systems and Applications, 59-62.

[51] Rob Raskin. 2003. The semantic web for Earth and environmental terminology (SWEET). In Proceedings of Workshop on Semantic Web Technologies for Searching and Retrieving Scientific Data.

[52] Robert G. Raskin. and Michael J. Pan. 2005. "Knowledge representation in Semantic Web for Earth and Environmental Terminology (SWEET)". Journal of Computers \& Geosciences, 1119-1125.

[53] Zaki Malik., Abdelmounaam Rezgui., Brahim Medjahed., Mourad Ouzzani., and A Krishna Sinha. 2010. "Semantic Integration in Geosciences", International Journal of Semantic Computing, 301-330.

[54] Viral Parekh., Jin-Ping Gwo., and Tim Finin. 2004. Ontology based Semantic Metadata for GeoScience Data. In Proceedings of International Conference on Information and Knowledge Engineering, 485-490.

[55] Klien,E., Lutz, M., and Kuhn,W. 2006. "Ontology-based discovery of geographic information services-An application in disaster management", Journal of Computers, Environment and Urban Systems, Elsevier, 102-123.

[56] Gang Liu., Yanni Wang., and Chonglong Wu. 2010. Research and Application of Geological Hazard Domain Ontology, In Proceedings of 18th International Conference on Geoinformatics , 1-6.

[57] Gomez-Perez, A., Ramirez, J., and Villazon-Terrazas, B. 2007. "An Ontology for Modelling Human Resources Management Based on Standards", Lecture Notes In Computer Science, 534-541, Springer-Verlag.

[58] Panceras Talita., Alvin W. Yeo., and Narayanan Kulathuramaiyer. 2010. Challenges in Building Domain Ontology for Minority Languages. In Proceedings of International Conference on Computer Applications and Industrial Electronics, 574 - 578.

[59] Saidah Saad., Naomie Salim., Hakim Zainal., and Zurina Muda. 2011. A process for building domain ontology: An experience in developing Solat ontology. In Proceedings of IEEE International Conference on Electrical Engineering and Informatics, 1-5.

[60] Rui Yao. and Jin Zhang. 2010. Design and Implementation of Chinese Ancient Poetry Learning System Based on Domain Ontology. In Proceedings of International Conference on e-Education, e-Business, eManagement and e-Learning, $460-463$.

[61] Fan Zhu., Apple W P Fok., Horace H S Ip., and Jiaheng Cao. 2008. ENGOnto: Integrated Multiple English
Learning Ontology for Personalized Education.In Proceeding of IEEE International Conference on Computer Science and Software Engineering, 210-213.

[62] Mao-sheng Lai . and Xiu-dan Yang. 2001. Ontologybased Metadata Schema for Chinese Digital Libraries. http://citeseerx.ist.psu.edu/viewdoc/summary?doi=10.1.1 .135 .7833 .

[63] Yi-hsing Chang. 2007. Automatically constructing a domain ontology for document classification. In Proceedings of the Sixth International Conference on Machine Learning and Cybernetics, 1942 - 1947.

[64] Luis Bermudez.John Graybeal., and Robert Arko. 2006. A Marine Platforms Ontology: Experiences and Lessons. In Proceedings of the Semantic Sensor Networks Workshop.

[65] Rommel N. Carvalho., Richard Haberlin., Paulo Cesar G. Costa., Kathryn, B. Laskey., and Chang, K.C. 2011. Modeling a Probabilistic Ontology for Maritime Domain Awareness. In Proceedings of the 14th International Conference Information Fusion, 1 - 8 .

[66] YUN Hongyan. 2009. Research on Building Ocean Domain Ontology. In Proceedings of Second International Workshop on Computer Science and Engineering, 146-150.

[67] Paul Witherell., Sundar Krishnamurty., Ian Grosse., and Jack Wileden. 2009. A Meronomic relatedness measure for domain ontologies using concept probability and multiset theory. In Proceedings of the 28th North American Fuzzy Information Processing Society Annual Conference.

[68] Ili Farhana Md Mahtar. and Nor Azan Mat Zin. 2010. Mathematical Knowledge Representation for Education Semantic Web based on Learning Style. In Proceeding of International Symposium on Information Technology.

[69] Harris, M.A., Clark,J., Ireland, A., Lomax, J., Ashburner, M., Foulger,R., Eilbeck,K., Lewis,S., Marshall,B., Mung all,C., Richter,J., Rubin,G.M., Blake,J.A., Bult,C., Dolan ,M.,Drabkin,H., Eppig,J.T., Hill,D.P., NiL.,Ringwald,M., Balakrishnan,R., Cherry,J.M., Christie,K.R., Costanzo, M.C., Dwight,S.S., Engel,S., Fisk,D.G., Hirschman,J.E., Hong,E.L.,Nash,R.S., Sethuraman,A., Theesfeld,C.L., Botstein,D., Dolinski, K., Feierbach, B., Berardini, T., Mundodi,S., Rhee,S.Y., Apweiler,R., Barrell,D., Camon, E., Dimmer,E., Lee,V., Chisholm,R., Gaudet,P., Kibbe, W., Kishore,R., Schwarz,E.M., Sternberg,P., Gwinn,M., Hannick,L., Wortman,J., Berriman, M., Wood, V., de la Cruz, N., Tonellato, P.,Jaiswal, P., Seigfried, T., White, R., and Gene Ontology Consortium. 2004. "The Gene Ontology (GO) database and informatics resource", Nucleic Acids Research. Oxford University Press.

[70] Melissa A Haendel., Georgios V Gkoutos., Suzanna E Lewis., and Christopher J Mungall. 2009. "Uberon: towards a comprehensive multi-species anatomy ontology", Nature Proceedings.

[71] Whetzel,P.L., Parkinson,H., Causton,H.C., Fan,L.,Fostel, J., Fragoso, G., Game, L., Heiskanen, M., Morrison, N., Rocca-Serra, P., Sansone, S.A., Taylor ,C., White, J., and Stoeckert,C.J.Jr. 2006.The MGED Ontology: a resource for semantics-based description of microarray experiments. Center for Bioinformatics and Department 
of Genetics, University of Pennsylvania School of Medicine, USA.

[72] Carol J.Bult., Janana T. Eppig., James A.Kadin., Joel E.Richardson., and Judith A. Balke. 2008. "The Mouse Genome Database (MGD): mouse biology and model systems", Nucleic Acids Research, Oxford University Press, D724-D728.

[73] Hoffman, H., Arnoldi, C., and Chuang, I. 2005. The Clinical Bioinformatics Ontology: A Curated Semantic Network Utilizing Refseq Information. In Proceedings of Pacific Symposium on Biocomputing.

[74] Charles E. Kahn., David S. Channin., and Daniel L. Rubin. 2006. "An Ontology for PACS Integration", Journal of Digital Imaging, 1-12.

[75] Werner Ceustersa., Barry Smithb., Anand Kumarb., and Christoffel Dhaena. 2004. "Ontology-Based Error Detection in SNOMED-CT". PubMed.482-6.

[76] Olivier Bodenreider. 2004. "The Unified Medical Language System (UMLS): Integrating biomedical terminology", Nucleic Acids Research, Oxford University Press, D267-D270.

[77] Zeeberg, B.R., Feng, W., Wang, G., Wang, M.D., Fojo, A.T., Sunshine,M., Narasimhan,S., Kane,D.W., Reinhold , W.C., Lababidi, S., Bussey, K.J., Riss, J., Barrett, J.C., and Weinstein, J.N .2003."GoMiner: a resource for biological interpretation of genomic and proteomic data". Journal of Genome Biology. BioMed Central. R28.

[78] Christopher J. O. Baker., René Witte., Arash ShabanNejad., Greg Butler., and Volker Haarslev. 2005. The Fungal Web Ontology: Application Scenarios. In Proceedings of Bioinformatics and Genomics $4^{\text {th }}$ International Semantic Web Conference.

[79] Arash Shaban-Nejad., Christopher J.O. Baker., Volker Haarslev., and Greg Butler. 2005. The Fungal Web Ontology: Semantic Web Challenges in Bioinformatics and Genomics, http://citeseerx.ist.psu.edu/ viewdoc/ download?doi=10.1.1.93.6919\&rep=rep1\&type=pdf.

[80] Nona Nader. and Rene Witte. 2010. Ontology-Based Extraction and Summarization of Protein Mutation Impact Information. In Proceedings of Workshop on Biomedical Natural Language Processing.

[81] Jaime Cantais., David Dominguez., Valeria Gigante., Loredana Laera., and Valentina Tamma. 2005. An example of food ontology for diabetes control. In Proceedings of Workshop on Ontology.

[82] Luciana V. Castilho., Heitor S. Lopes., Cesar A. Tacla. 2008. Modeling and Building an Ontology for Neuropediatric Physiotherapy Domain. In Proceedings of Eighth International Conference on Hybrid Intelligent Systems, $210-215$.

[83] Lazzari,C.De., Guerrieri,E., and Pisanelli, D.M. 2003. A Domain Ontology for Mechanical Circulatory Support Systems. In Proceedings of IEEE Computers in Cardiology, $417-419$.

[84] Jan Paralic. and Ivan Kostial. 2003. Ontology-based Information Retrieval. In Proceedings of 14th International Conference on Information and Intelligent systems, $23-28$.
[85] Clement Jonquet., Paea LePendu., Sean M. Falconer., Adrien Coulet., Natalya F. Noy., Mark A. Musen., and Nigam H. Shah. 2011." NCBO Resource Index: Ontology-Based Search and Mining of Biomedical Resources", Web Semantics: Science, Services and Agents on the World Wide Web,Elsevier, 316-324.

[86] Jennifer Golbeck., Gilberto Fragoso., Frank Hartel., Jim Hendler., Jim Oberthaler., and Bijan Parsia. 2003." The National Cancer Institute's Thésaurus and Ontology", Journal of Web Semantics: Science, Services and Agents on the World Wide Web, Elsevier, 75-80.

[87] Landon T. Detwiler., Emily Chung., Ann Li., José L.V. Mejino Jr., Augusto V. Agoncillo., James F. Brinkley., Cornelius Rosse., and Linda G. Shapiro., 2004. "A Relation-Centric Query Engine for the Foundational Model of Anatomy",PubMed,Structural Informatics Group publication, 341-5.

[88] Mei-ying Jia., Bing-ru Yang., De-quan Zheng., and Weicong Sun. 2009. Research on Domain Ontology Construction in Military Intelligence. In Proceedings of Third International Symposium Intelligent Information Technology Application, 116-119.

[89] Liu Yang., Zhigang Hu., Jun Long., and Tao Guo., 2011. 5W1H-based Conceptual Modeling Framework for Domain Ontology and its Application on STOP. In Proceedings of IEEE Seventh International Conference on Semantics, Knowledge and Grids.203-206.

[90] Song Jun-feng., Zhang Wei-Ming., Xiao Wei-dong., and $\mathrm{Xu}$ Zhen-ning., 2005.Study on construction and integration of military domain ontology situation ontology and military rule ontology for network centric warfare. In Proceedings of IEEE International Conference Publication on e-Technology, e-Commerce and e-Service, $368-373$.

[91] Laban, S., Desouky, A.I.EI., ElHefnawy,A.S., and ElGebaly, A.F. 2010. Ontology Potentials in Increasing Effectiveness of Safeguards and Nuclear weapon Nonproliferation Activities. In Proceedings of Symposium on International Safeguards. Preparing for Future Verification Challenges, IAEA conference.

[92] Junfang Shi. and Li Liu., 2010. Web information extraction based on news domain ontology Theory. In Proceedings of IEEE 2nd Symposium on Web Society, 416-419.

[93] Zongxiao Yang., Chuanye Cheng., and Zhiqiang Feng. 2008. Construction of Ontology-based Safety Assessment System for Power Plants. In Proceedings of IEEE International Conference on Networking, Sensing and Control, 1092-1096.

[94] Khadir, M.T. and Klai, S. 2010. A steam turbine diagnostic maintenance system based on evaluative domain ontology. In Proceedings of International Conference on Machine and Web Intelligence, 360-367.

[95] Munir Merdan., Gottfried Koppensteiner., Ingo Hegny., and Bernard Favre-Bulle. 2008. Application of an Ontology in a Transport Domain. In Proceedings of IEEE International Conference on Industrial Technology. $1-6$. 